\title{
Winning of High Purity Iron from Red Mud and Iron Concentrated Tailing
}

\author{
By M. Isshiki* and K. Igaki*
}

\begin{abstract}
Winning of high purity iron was carried out from red mud in aluminum refinery and iron concentrated tailing in copper smelting by leaching with hydrochloric acid and anion exchange separation. The iron obtained was further purified by floating zone-melting in vacuum. The purity of the iron refined was examined by measuring $R R R_{H}$ values and by analytical methods such as activation analysis and spark source mass spectroscopic analysis. It is confirmed that the iron purified is very pure and nearly corresponds to the commercial high purity iron such as JohnsonMatthey iron with nominal purity of $99.99 \%$. In order to know the chemical composition of the residue after leaching, PIXE method was applied.
\end{abstract}

(Received January 10, 1981)

\section{Introduction}

Nowadays, it becomes very important to utilize the resource effectively, because many natural resources have approached to the nearly exhausted state. Since some kinds of industrial by-products can be used as a resource for materials of practical value, effective use of industrial by-products should be inquired, especially in avoiding the environmental pollution.

Red mud in aluminum refinery and iron concentrated tailing in copper smelting have been considered as the promising source of iron. Although many studies have been made to develop the process to utilize them, few process has been put to practical use because of its unfavorable economical situation.

It has been developed the preparation process of high purity iron ${ }^{(1)(2)}$ by the anion exchange method in a medium of hydrochloric acid solution. This process can be easily applied to the winning of iron from red mud and iron concentrated tailing. If iron of nearly the same purity as the commercial grade is obtained, this process will be confirmed to be very effective.

* Department of Materials Science, Faculty of Engineering, Tohoku University.

\section{Experimental}

\section{Winning of iron}

The process for winning of high purity iron is shown in Fig. 1. Leaching of red mud or iron concentrated tailing was carried out in the solution of $9 \mathrm{kmol} / \mathrm{m}^{3}(9 \mathrm{~N})-\mathrm{HCl}$ using a water bath. The operation of anion exchange separation consists of three steps, fixing Fe(III) on resin in $9 \mathrm{kmol} / \mathrm{m}^{3}-\mathrm{HCl}$ for $2 \mathrm{~h}$, washing out

\author{
Starting materials \\ I \\ Leaching with $9 \mathrm{~N}-\mathrm{HCl}$<smiles>[CH]</smiles> \\ Filtration \\ Anion exchange separation \\ I \\ Purified $\mathrm{FeCl}_{3}$ solution \\ Evaporation to dryness<smiles>[Tl]</smiles> \\ Oxidation \\ Reduction with wet hydrogen<smiles>[CH]1CC1</smiles> \\ Floating zone refining<smiles>C1=CC=C1</smiles> \\ Wet hydrogen treatment
}

Fig. 1 Winning process of high purity iron. 
of impurities remaining in the space among resin by $5 \mathrm{kmol} / \mathrm{m}^{3}-\mathrm{HCl}$ for $2.5 \mathrm{~h}$ or by 6 $\mathrm{kmol} / \mathrm{m}^{3}-\mathrm{HCl}$ for $3 \mathrm{~h}$ on red mud or iron concentrated tailing, respectively. In order to remove $\mathrm{Cu}$ effectively, $6 \mathrm{kmol} / \mathrm{m}^{3}-\mathrm{HCl}$ was used in the case of iron concentrated tailing. Elution of $\mathrm{Fe}$ (III) was carried out with 1 $\mathrm{kmol} / \mathrm{m}^{3}-\mathrm{HCl}$. The flow rate of these solution was fixed at $7.5 \times 10^{-7} \mathrm{~m}^{3} / \mathrm{s}$. Strong base anion exchange resin Diaion SA10B was used as packed into the column made of acrylic acid resin with the length and diameter of 1000 and $70 \mathrm{~mm}$.

Purified ferric chloride solution was evaporated to dryness and oxidized in air. Iron oxide was reduced to the metallic state and sintered by wet hydrogen at $800^{\circ} \mathrm{C}$. The sponge iron obtained was melted and formed to a rod shape of about $5 \mathrm{~mm}$ diameter in an atmosphere of dry hydrogen. Floating zone-refining was carried out in vacuum $\left(2 \times 10^{-3} \mathrm{~Pa}\right)$. The purity of iron was estimated from the value of residual resistivity ratio in a longitudinal magnetic field $\left(R R R_{\mathrm{H}}\right)$ and by several analytical methods.

\section{Composition analysis}

Spark source mass spectroscopic analysis, activation analysis and proton induced X-ray emission (PIXE) were applied to the examination of the distribution of the elements at each step of the winning process. Short refer only to the PIXE method will be given in this paper, since others are well known and the detail of the activation analysis is already reported elsewhere ${ }^{(3)}$.

As well known, bombardment of charged particles to materials causes the excitation. Analysis is performed by measurement of the $\mathrm{X}$-ray spectrum through the excitation. When the sample is thin enough not to decrease the energy of incident protons $\left(E_{p}\right)$, detected counts of $K$ series $\mathrm{X}$-ray from $i$ element $\left(C_{i}^{K}\right)$ are expressed by

$$
C_{i}^{K}=I \cdot \Omega \cdot E_{f f} \cdot F_{m} \cdot F_{a} \cdot F_{s} \cdot \sigma_{i}^{K}\left(E_{p}\right) \cdot N_{i},
$$

where $I, \Omega$ and $E_{f f}$ is the flux of the incident beam, the solid angle against the detector and the detection efficiency of the $\mathrm{Si}(\mathrm{Li})$ detector, respectively. $S_{m}, F_{a}$ and $F_{s}$ are the factors for correction of absorption through a mylar window of the sample chamber, the air pass and the sample. $\sigma_{i}^{K}\left(E_{p}\right)$ and $N_{i}$ is the cross section for excitation of the $K$-shell of the $i$-element at $E_{p}$ and the number of $i$-atoms, respectively. $N_{i}$ can be calculated from the measured $C_{i}^{K}$ using the values of the factors in eq. (1) determined for the experimental system and conditions.

If the sample is very thin, $F_{s}$ will be close to unity, but the correction is necessary judging from the diameter of the powder specimens. Assuming that each grain is sphere, the mean thickness $P$ was estimated from the radius of sphere $r_{m}$ as $P=\left(\pi r_{m}\right) / 2$. For the mean thickness $P, F_{s}$ is calculated as

$$
\begin{aligned}
F_{s} & =\int_{0}^{P} \exp (-\mu \rho x) d x / \int_{0}^{P} d x \\
& =[1-\exp (-\mu \rho P)] /(\mu \rho P),
\end{aligned}
$$

where $\mu$ and $\rho$ are the mass absorption coefficient and the density of the sample. As well known, the mass absorption coefficient is expressed as $\rho^{-1} \sum_{j} c_{j} \mu_{j}^{i}$, where $c_{j}$ and $\mu_{j}^{i}$ are the concentration of the constituent element $j$ and the mass absorption coefficient of the $j$ element for $K \mathrm{X}$-rays emitted from the $i$ element, respectively.

Since the real values of $c_{j}$ are unknown at first, $F_{s}$ cannot be determined. As the zeroth approximation, $c_{j}^{0}$ is estimated from the peak area of the detected constituent elements after correction except $F_{s}$. Using $c_{j}^{0}, F_{s}^{1}$ is calculated by eq. (2) taking into consideration the absorption effect by the main six metallic elements and oxygen with the corresponding concentration in the usual chemical oxide formula. Applying correction using $F_{s}^{1}, c_{j}^{1}$ is calculated. The correction by $F_{s}$ was iterated until the obtained values converged.

The densities of the samples were measured by means of Archimedes' method. Grain size of the samples was determined from the observation with a scanning electron microscope.

The energy and current of the proton beam is $3 \mathrm{MeV}$ and about $1 \mathrm{nA}$, respectively. Mylar film with $10^{-5} \mathrm{~m}$ thickness was used as backing. Experiments were performed at the Cyclotron Radioisotope Center of Tohoku University. 


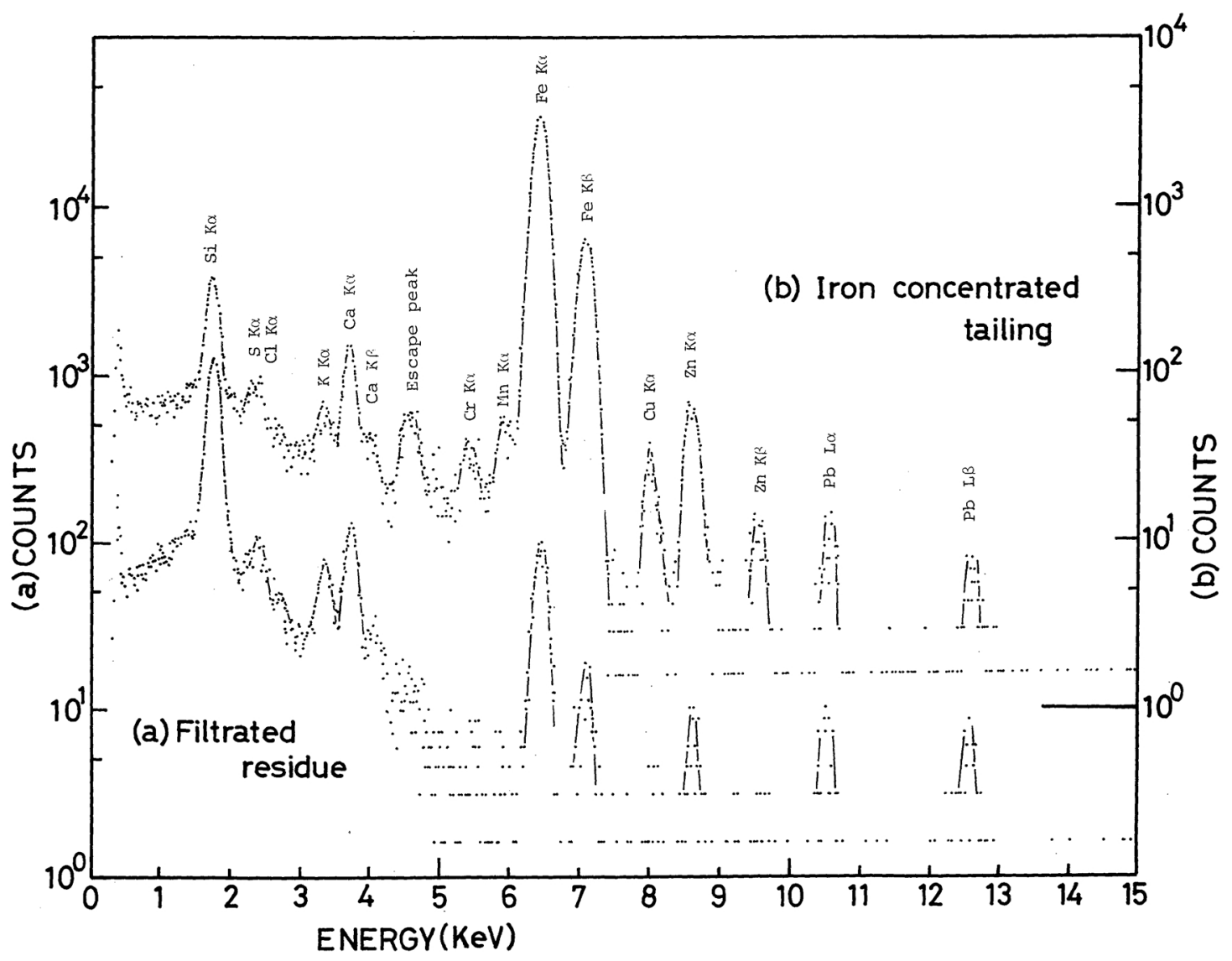

Fig. 2 X-ray spectra of iron concentrated tailing and filtrated residue.

Table 1 Density and grain size of the samples.

\begin{tabular}{ccccc}
\hline Sample & Red mud & $\begin{array}{c}\text { Filtrated residue } \\
\text { of red mud }\end{array}$ & $\begin{array}{c}\text { Iron concentrated } \\
\text { tailing }\end{array}$ & $\begin{array}{c}\text { Filtrated residue of } \\
\text { iron concentrated tailing }\end{array}$ \\
\hline Grain size $\left(10^{-6} \mathrm{~m}\right)$ & $20 \pm 10$ & $7 \pm 3$ & $20 \pm 10$ & $4 \pm 2$ \\
\hline Density $\left(10^{3} \mathrm{~kg} / \mathrm{m}^{3}\right)$ & 3.41 & 2.78 & 4.26 & 2.40 \\
\hline
\end{tabular}

\section{Results}

X-ray spectra of iron concentrated tailing and the filtrated residue after leaching are shown in Fig. 2. The density and grain size of the samples are given in Table 1. Figure 3 shows the typical relation between the normalized peak area corrected by $F_{s}^{n}$ and the number of iteration $n$. It is known that iteration more than three times gives a fixed value. The analytical results are shown in Table 2 . From the results of Table 2, it is known that iron is the main element extracted in the leaching process.

Floating zone-refining was carried out on the iron purified by the anion exchange method. The values of $R R R_{\mathrm{H}}$ and the concentrations of impurities are shown in Fig. 4 and Table 3.

It is found that the iron winned from red 
mud is very pure and the obtained purity nearly corresponds to the commercial high purity iron such as Johnson-Matthey iron with a nominal purity of $99.99 \%$. The iron winned from the iron concentrated tailing is purer than the commercial grade electrolytic iron, though less pure than that from red mud.

\section{Discussion}

It is suggested from the results shown in Fig. 3 that special attention must be paid to the dimension or shape of the samples in order

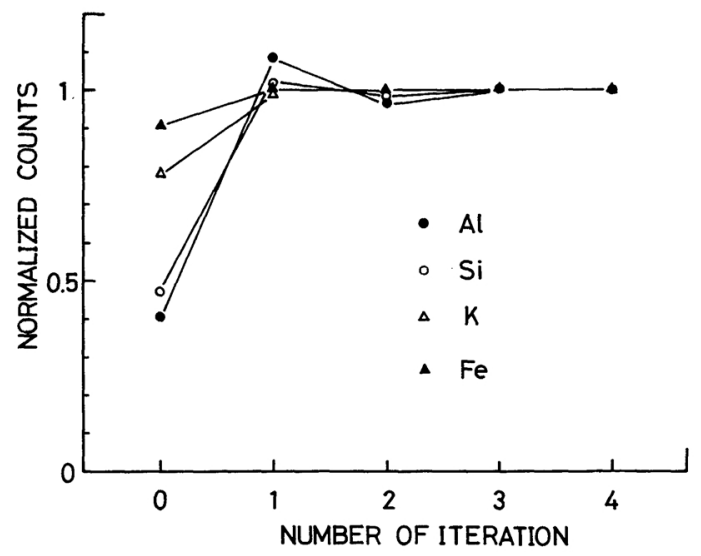

Fig. 3 Typical relation between the number of iteration of correction by the absorption through sample and X-ray peak area normalized by that of 4 th iteration on filtrated residue of red mud. to carry out quantitative analysis from the X-ray spectrum on PIXE method, because the absorption of X-ray, especially, low energy $\mathrm{X}$-ray from light elements is strongly dependent of the path in the sample.

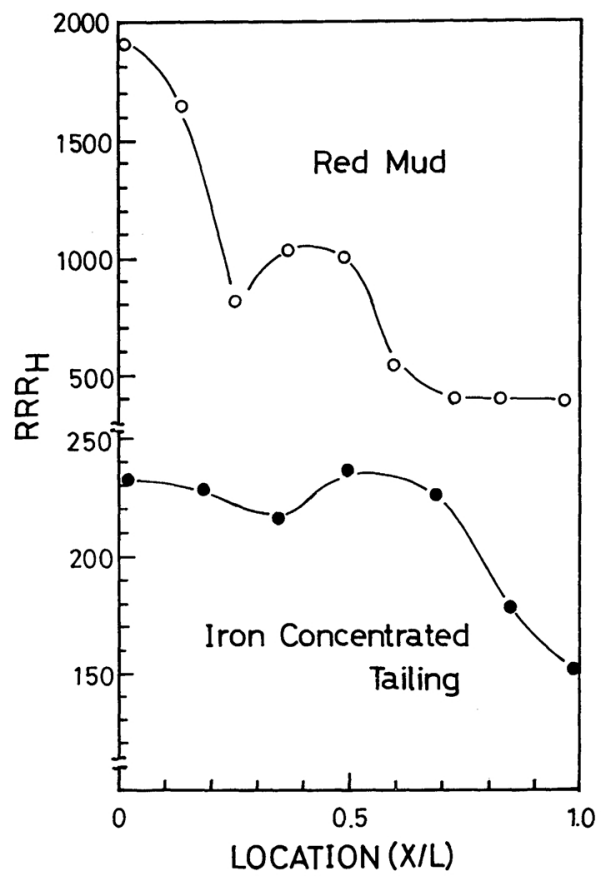

Fig. 4 RRR $_{H}$ profiles of floating zone-refined irons obtained from red mud and iron concentrated tailing. Two times anion exchange separation were performed in the case of red mud.

Table 2 Composition /in mass \%) of starting materials and filtrated residues of red mud and iron concentrated tailing.

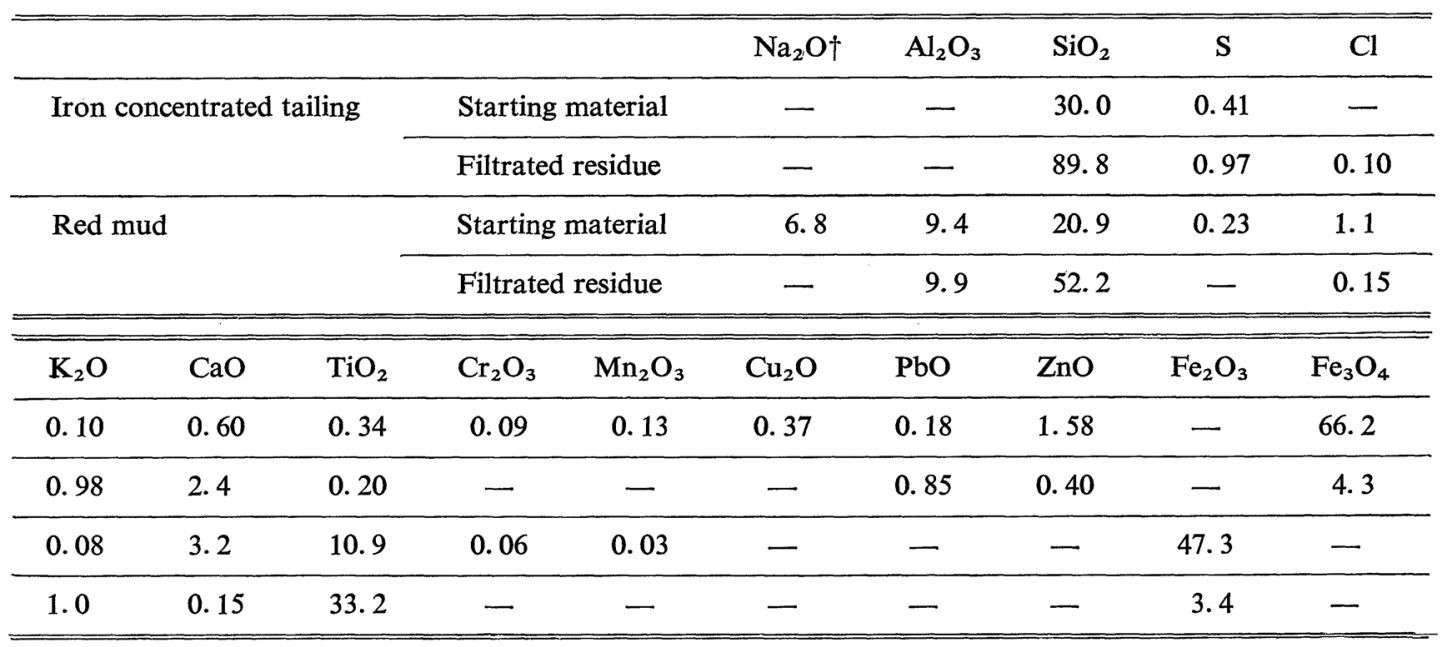

$\nmid$ Result of $\mathrm{Na}_{2} \mathrm{O}$ is the data given by supplier. 
Table 3 Analytical results (in mass ppm) of the iron winned by neutron activation analysis* (AA) and mass spectroscopic analysis** (MSA). III indicates the operation of anion exchange separation in the state of Fe(III). Typical results of commercial pure iron of nominal purity $99.99 \%$ supplied by Johnson-Matthey Co. is also shown.

\begin{tabular}{|c|c|c|c|c|c|c|c|}
\hline \multirow{3}{*}{$\begin{array}{c}\text { Sample } \\
\text { Purification } \\
\text { Method }\end{array}$} & \multicolumn{3}{|c|}{ Red mud } & \multicolumn{2}{|c|}{ Iron concentrated tailing } & \multirow{2}{*}{\multicolumn{2}{|c|}{ J-M Iron }} \\
\hline & \multirow{2}{*}{$\frac{\mathrm{III} \times 2}{\mathrm{AA}}$} & \multicolumn{2}{|c|}{$\mathrm{III} \times 2+5 \mathrm{ZM}$} & \multirow{2}{*}{$\frac{\mathrm{III} \times 1}{\mathrm{AA}}$} & \multirow{2}{*}{$\frac{\mathrm{II} \times 1+5 \mathrm{ZM}}{\mathrm{AA}}$} & & \\
\hline & & MSA & $\mathrm{AA}$ & & & MSA & $\mathrm{AA}$ \\
\hline $\mathrm{Na}$ & $<1$ & 4.0 & 0.64 & 3.2 & 5.1 & 12 & $<0.6$ \\
\hline $\mathrm{Mg}$ & & 0.2 & & & & 1.6 & \\
\hline $\mathrm{Al}$ & & ND & & & & 0.44 & \\
\hline $\mathrm{Si}$ & & 5.0 & & & & 1.5 & \\
\hline $\mathbf{P}$ & & 0.2 & & & & 0.5 & \\
\hline $\mathbf{S}$ & & 0.5 & & & & 1.8 & \\
\hline $\mathrm{Cl}$ & & 1.0 & & & & 0.7 & \\
\hline $\mathbf{K}$ & & 3.0 & & & & 2.2 & \\
\hline $\mathrm{Ca}$ & & 0.3 & & & & 2.2 & \\
\hline $\mathrm{Ti}$ & & 0.1 & & & & 1.0 & \\
\hline V & & 0.1 & & & & 0.02 & \\
\hline $\mathrm{Cr}$ & & 1.0 & & & & 3.3 & \\
\hline $\mathrm{Mn}$ & $<0.4$ & 3.0 & $<0.1$ & 0.3 & $<0.2$ & 3.0 & 3.7 \\
\hline $\mathrm{Co}$ & & 0.3 & & & & 0.09 & \\
\hline $\mathrm{Ni}$ & & 1.0 & & & & 0.3 & \\
\hline $\mathrm{Cu}$ & 1.5 & 1.5 & $<0.5$ & 24.6 & 0.16 & 1.5 & 4.8 \\
\hline $\mathrm{Zn}$ & & 0.7 & & & & $<0.002$ & \\
\hline $\mathrm{Ga}$ & 78 & 2.0 & $<0.2$ & 11 & $<0.2$ & 0.1 & $<2$ \\
\hline As & $<1.5$ & 0.4 & $<0.8$ & 1 & $<0.2$ & 0.1 & $<0.8$ \\
\hline $\mathrm{Nb}$ & & 0.5 & & & & 0.1 & \\
\hline Mo & & 2.0 & & & & 0.2 & \\
\hline $\mathrm{Ag}$ & & 0.1 & & & & 0.03 & \\
\hline $\mathrm{Ta}$ & & 0.1 & & & & 0.09 & \\
\hline W & $<3$ & 0.3 & $<1.2$ & 4 & 3.3 & ND & $<0.8$ \\
\hline $\mathrm{Au}$ & & ND & & 0.03 & $<0.004$ & ND & 0.49 \\
\hline $\mathrm{Pb}$ & & 0.06 & & & & ND & \\
\hline
\end{tabular}

* Activation analysis was done under the Visiting Researchers Program of Kyoto University Reactor Research Institute.

** Mass spectroscopic analysis was cordially performed by the aid of Sumitomo Electric Works.

Pure iron nearly in the same grade as the commercial grade high purity iron was obtained from red mud and iron concentrated tailing. This fact indicates that the purification process developed previously ${ }^{(1)}$ is very effective and gives materials of commercial value from the industrial by-products. The most important point to make this process practically available is the development of a method for cyclic utilization of hydrochloric acid.

\section{Acknowledgment}

The authors wish to express their gratitude to the members of Cyclotron Radioisotope Center of Tohoku University and Research Reactor Institute of Kyoto University.

\section{REFERENCES}

(1) M. Isshiki and K. Igaki: Trans JM, 18 (1977), 412.

(2) M. Isshiki and K. Igaki: Tech. Rep. Tohoku Univ., 44 (1979), 331.

(3) M. Isshiki, H. Nitta, Y. Noda, S. Itoh, K. Igaki, A. Mizohata, T. Mamuro, T. Tsujimoto and S. Iwata: Radioisotopes, 28 (1979), 3. 